\title{
Acupuncture therapy in treating migraine: results of a magnetic resonance spectroscopy imaging study
}

This article was published in the following Dove Press journal: Journal of Pain Research

\author{
Tao $\mathrm{Gu}^{1,2}$ \\ Lei $\operatorname{Lin}^{3}$ \\ Yun Jiang ${ }^{4}$ \\ Juan Chen' \\ Ryan CN D'Arcy ${ }^{2,5,6}$ \\ Min Chen' \\ Xiaowei Song ${ }^{2,5,6}$ \\ 'Department of Radiology, Beijing \\ Hospital, National Center of \\ Gerontology, Beijing, China; ${ }^{2}$ Simon \\ Fraser University Image Tech \\ Laboratory, Surrey Memorial Hospital, \\ Surrey, BC, Canada; ${ }^{3}$ Department \\ of Acupuncture and Moxibustion, \\ Beijing Hospital, National Centre \\ of Gerontology, Beijing, China; \\ ${ }^{4}$ Department of Neurology, \\ Beijing Hospital, National Centre \\ of Gerontology, Beijing, China; \\ ${ }^{5}$ Department of Applied Sciences and \\ Computing Science, Simon Fraser \\ University, Burnaby, BC, Canada; \\ ${ }^{6} \mathrm{Health}$ Sciences and Innovation, \\ Surrey Memorial Hospital, Fraser \\ Health Authority, Surrey, BC, Canada
}

Correspondence: Xiaowei Song Health Sciences and Innovation, Surrey Memorial Hospital, Fraser Health Authority, I3750 96th Avenue, Surrey, British Columbia V3V IZ2, Canada $\mathrm{Tel}+\mathrm{I} 6045855666 \sim 774986$ Email Xiaowei.song@fraserhealth.ca

Min Chen

Department of Radiology, Beijing Hospital, National Center of Gerontology, \#I Dahua Road, Dongdan, Beijing 100730, China

Tel +86 I0 85I3 3804

Email cjr.chenmin@vip.163.com
Background: Acupuncture has been proven to be effective as an alternative therapy in treating migraine, but the pathophysiological mechanisms of the treatment remain unclear. This study investigated possible neurochemical responses to acupuncture treatment.

Patients and methods: Proton magnetic resonance spectroscopy imaging was used to investigate biochemical levels pre- and post-acupuncture treatment. Participants $(\mathrm{N}=45)$ included subjects diagnosed with: 1) migraine without aura; 2) cervicogenic headache; and 3) healthy controls. Participants in the two patient groups received verum acupuncture using acupoints that target migraine without aura but not cervicogenic headache, while the healthy controls received a sham treatment. All participants had magnetic resonance spectroscopy scans before and after the acupuncture therapy. Levels of brain metabolites were examined in relation to clinical headache assessment scores.

Results: A significant increase in $\mathrm{N}$-acetylaspartate/creatine was observed in bilateral thalamus in migraine without aura after the acupuncture treatment, which was significantly correlated with the headache intensity score.

Conclusion: The data demonstrate brain biochemical changes underlying the effect of acupuncture treatment of migraine.

Keywords: acupuncture, metabolites, migraine, magnetic resonance spectroscopy imaging, brain, pain transmission pathways

\section{Introduction}

Migraine is a common headache disorder. The World Health Organization ranks migraine very high among disability-causing disease worldwide. ${ }^{1}$ Therapeutic treatment of migraine largely relies on medications, ${ }^{2,3}$ but can also involve nonpharmacological therapies, for enhancing medication effect especially in patients who are less responsive. ${ }^{4}$ The effect of acupuncture in the treatment of migraine has been well recognized in Traditional Chinese Medicine (TCM), and, in recent years, also in modern Western medicine: Meta-analysis using the Cochrane database suggests that acupuncture on migraine prophylaxis is both safe and effective, without major side adverse effects. ${ }^{5,6}$ Accordingly, it is recommended that acupuncture can be used as an alternative therapy in migraine patients willing to undergo this treatment. ${ }^{5,6}$

The physiological mechanism of acupuncture treatment of migraine is unclear. Research has suggested changes in the nervous system performance in response to acupuncture treatment, including changes in the concentrations of $\mathrm{K}^{+}, \mathrm{Na}^{+}$, and $\mathrm{Ca}^{+}$ in the neurons. ${ }^{7,8}$ Several hypotheses have been proposed that emphasize the impact 
of acupuncture on relieving pain, including eliciting an analgesic effect on the hypothalamic-pituitary-adrenal axis and the endogenous opioid system. ${ }^{9}$

It is well known that the thalami play an important role in sensory pain transmission pathways. ${ }^{10}$ Anatomical investigations have repeatedly shown that information of pain stimulus is typically transmitted from the sensory organs via thalamic sites to a cerebral cortical area such as the primary somatosensory cortex and the anterior cingulate cortex (which is associated with emotion control). ${ }^{11,12}$ Neurons in these brain regions exhibit monotonic increases to increasing pain stimulus intensities. ${ }^{12}$ However, it is unclear if and how the application of acupuncture intervention in treating migraine affects this pain pathway.

Proton magnetic resonance spectroscopy imaging (MRSI) can be used to address this gap. MRSI is an advanced noninvasive imaging method that allows for quantification of in vivo tissue metabolism in localized brain regions by exploiting the magnetic properties of the hydrogen nuclei. ${ }^{13}$ The technique has been used to investigate brain metabolism in a wide range of disorders. In migraine, altered brain metabolite levels, notably a decrease in $\mathrm{N}$-acetylaspartate (NAA, a putative marker of neural functionality) have been observed. ${ }^{14,15}$ Only a limited number of studies have investigated the effect of acupuncture using MR spectroscopy, while most of which have focused on metabolism-related disorders such as obesity and fibromyalgia. ${ }^{16,17}$ To date, no study has investigated the changes of brain metabolites in response to acupuncture therapy on migraine using MRSI technology.

In the present study, we investigated whether neurochemical changes are associated with the acupuncture treatment in regulating the function of pain transmission pathways. We hypothesized that the thalami (TH), primary somatosensory cortex (S1), and anterior cingulate cortex (ACC), which are important in the transmission of pain, may be variably affected by the acupuncture treatment, and that such effect could be revealed from the changes in key brain metabolites such as NAA, creatine $(\mathrm{Cr}$, indicator of the energy metabolism), and choline-containing compounds (Cho, marker of the membrane turnover). Our primary objective was to detect changes in NAA, Cr, and Cho in brain regions of the $\mathrm{TH}, \mathrm{S} 1$, and $\mathrm{ACC}$ in association with the acupuncture treatment of migraine. To understand the clinical mindfulness of the neurochemical changes, we also examined the relationship of the level of the metabolites with clinical outcomes.

\section{Patients and methods}

\section{Participants}

Three groups of participants including: 1) patients with migraine without aura (MwoA; $\mathrm{n}=15$ ); 2) patients with cervicogenic headache $(\mathrm{CH} ; \mathrm{n}=15)$; and 3$)$ healthy control subjects (HC; $n=15$ ), were recruited at Beijing Hospital (for MwoA and $\mathrm{CH}$ ) and Beijing University of Traditional Chinese Medicine (for HC). Participants were adults with a female to male ratio of 2:1 (according to the higher prevalence of migraine syndromes in women in the general population), ${ }^{18}$ aged between 18 and 60 years, right-handed, neither had taken any prophylactic headache medicine nor had preventive acupuncture treatment during the past three months prior to study enrollment. Each subject underwent a clinical assessment that consisted of a medical history evaluation, a physical examination, hepatic and renal function tests, and routine analysis of blood, urine, and stool. The patient participants must have had a minimum of 6 months of headache history and a minimum of one headache attack per month over the past 3 months. Volunteers were excluded if they were alcoholic or drug abusers, pregnant or lactating, had a history of or suffered from any psychiatric, neurological, cardiovascular, respiratory, or renal illnesses, or had been diagnosed with other comorbidities. Volunteers also underwent a standard MRI safety screening and an acupuncture safety checking; those with any MRI safety or any acupuncture safety contraindications (e.g. a bleeding tendency) and those who preferred to take any medications for treating headache during the period of the study were also excluded. All subjects had a routine clinical anatomical MRI within a week of the enrolment and those with any incidental findings (e.g. brain tumor, cerebrovascular disease, etc) were also excluded.

Migraine diagnoses were made based on the International Classification of Headache Disorders, 2nd edition (ICHD-II) criteria. ${ }^{1} \mathrm{MwoA}$ is highly prevalent globally and is defined as a syndrome characterized by recurrent attacks of throbbing unilateral headache, autonomic nervous system dysfunction, without neurological transient symptoms (migraineous aura). Here, all MwoA patients met the criteria as having the "Gang Yang Shang Kang", i.e. meaning liver meridian hyperactivity based on TCM. ${ }^{19} \mathrm{CH}$ is also often seen, although not as commonly as $\mathrm{MwoA}^{20}$ and is defined as a syndrome characterized by chronic hemicranial pain that is referred to the head from either bony structure or soft tissues of the neck. $\mathrm{CH}$ was chosen as the reference condition because it can present characteristics similar to those of MwoA. ${ }^{20}$ Importantly, although MwoA and $\mathrm{CH}$ can sometimes present with similar 
symptoms, each of the syndromes has a different mechanism and thus different primary acupoints for effective acupuncture treatment. ${ }^{21,22}$

\section{Experiment}

Data collection took 9 weeks to complete (Table 1). Baseline data (e.g. clinical headache assessments) were collected at the end of week 4, post the initial enrollment. Acupuncture intervention was carried out during week 5 , during which all participants received either a verum (MwoA \& $\mathrm{CH})$ or a sham acupuncture intervention (HC) on a daily basis (see below for details). Post-treatment data collection occurred at the end of week 9. Each subject had two MRSI sessions (in addition to the initial anatomical MRI scan); one at the end of week 4 (i.e. immediately after the baseline data collection) and the other at the end of week 5 (i.e. after ending the whole acupuncture session).

\section{Acupuncture intervention}

The acupuncture treatment consisted of five sessions; i.e. one session per day from Monday through Friday during the treatment week.

The same acupoints were used for the MwoA and the $\mathrm{CH}$ subjects. The choice of these acupoints targeted effective treatment for MwoA based on TCM practice guide ${ }^{19}$ (i.e. the acupoints are along the targeted meridians for treating "Gang Yang Shang Kang” MwoA, which are different from the primary therapeutic acupoints for $\mathrm{CH}^{21,22}$ ). These acupoints included "Baihui" (DU20) on the top of the head, bilateral "Fengchi" (GB20) on the neck, and bilateral "Xingjian" (LR2) on the dorsum of each foot. These are standard acupoints that are always used in the clinical acupuncture therapy of MwoA. ${ }^{23}$ In the $\mathrm{HC}$ group, the sham acupuncture was administered at non-acupoints (located $10 \mathrm{~mm}$ straight medial from the targeted acupoints), to rule out the possibility of pain conduction due to any acupuncture procedure-induced stimulation, if the procedure was applied at an acupoint. ${ }^{24}$

All verum and sham acupuncture treatments were performed by a practitioner with 7 years of clinical experience. Acupuncture was performed following standardized clinical acupuncture intervention procedures (i.e. the disposable stainless steel acupuncture needles $(0.25 \times 40 \mathrm{~mm}$; Ande Co., Guizhou, China) were inserted into the point with an angled insertion of $1.5-2.5 \mathrm{~cm}$; twirling, lifting, and thrusting to obtain the "De Qi" sensation, connecting the needle at points with an electric acupuncture apparatus (G6805-I type, QingDao XinSheng Industrial Co., Ltd, QingDao, China) with dilatational wave lasting for 30 minutes. Stimulus intensity depended on the patient's tolerance level).

\section{Clinical measures}

Clinical pain measures included headache intensity, headache frequency, and duration of each headache attack over the past 4 weeks at baseline and at follow-up, and were used to assess

Table I Experimental design

\begin{tabular}{|c|c|c|c|c|}
\hline Experiment design & Group & Migraine without aura & Cervicogenic headache & Healthy control \\
\hline & $\begin{array}{l}\text { Sample size } \\
\text { (female/male) }\end{array}$ & $10 / 5$ & $10 / 5$ & $10 / 5$ \\
\hline Clinical assessment & Headache intensity & \multicolumn{3}{|c|}{ On visual analog scores (0: no pain to 10: worst pain) } \\
\hline$($ week $1-4)$ & Headache frequency & \multicolumn{3}{|c|}{ The number of headaches separated by $48+$ hours of pain-free intervals within the past 4 weeks } \\
\hline & Duration of headache & \multicolumn{3}{|c|}{ The time in hours from the beginning till the end of a headache episode } \\
\hline MRS test & Sequence & \multicolumn{3}{|c|}{ 2D-multiple voxel PRESS } \\
\hline at the end of week 4 & Location & \multicolumn{3}{|c|}{ Left thalamus, right thalamus, anterior cingulate cortex and posterior part of paracentral gyrus } \\
\hline & Metabolite ratio & \multicolumn{3}{|c|}{ NAA/Cr, Cho/Cr, Cho/NAA } \\
\hline Intervention therapy & Frequency & \multicolumn{3}{|l|}{ I time/day for 5 days } \\
\hline (week 5) & Method & \multicolumn{3}{|l|}{ Acupuncture } \\
\hline & Acupoints & \multicolumn{3}{|l|}{ Verum* or sham** } \\
\hline MRS test & Sequence & \multicolumn{3}{|l|}{ 2D-multiple voxel PRESS } \\
\hline at the end of week 5 & Location & \multicolumn{3}{|c|}{ Left thalamus, right thalamus, anterior cingulate cortex and posterior part of paracentral gyrus } \\
\hline & Metabolite ratio & \multicolumn{3}{|l|}{$\mathrm{NAA} / \mathrm{Cr}, \mathrm{Cho} / \mathrm{Cr}, \mathrm{Cho} / \mathrm{NAA}$} \\
\hline Clinical assessment & Headache intensity & \multicolumn{3}{|c|}{ On visual analog scores (0: no pain to 10: worst pain) } \\
\hline (week 6-9) & Headache frequency & \multicolumn{3}{|c|}{ The number of headaches separated by $48+$ hours of pain-free intervals within the past 4 weeks } \\
\hline & Duration of headache & \multicolumn{3}{|c|}{ The time in hours from the beginning till the end of a headache episode } \\
\hline
\end{tabular}

Note: *Baihui (DU20), bilateral Fengchi (GB20) and Xingjian (LR2) for patients of both groups. **10 mm straight medial from the Baihui (DU20), bilateral Fengchi (GB20) and Xingjian (LR2) for healthy controls.

Abbreviations: MRS, magnetic resonance spectroscopy; PRESS, single-voxel point-resolved spectroscopy; NAA, N-acetylaspartate; Cr, creatine; Cho, choline-containing compounds. 
the efficacy of acupuncture treatment. Headache intensity was evaluated with a $0-10$ visual analog scale (VAS), with 0 indicating no pain, 10 indicating the worst pain imaginable, and so with the intermediate values incrementally. ${ }^{25}$ The frequency of headache attacks was defined as the number of headache attacks separated by pain-free intervals of at least 48 hours within the past 4 weeks. The duration of headache attacks was defined as the time in hours, from the beginning until the end of a headache episode. In the case where a patient fell asleep with the pain episode, the end of the headache episode was considered as 24 hours.

\section{MR spectroscopy data acquisition}

MR spectroscopy data acquisition used a Whole-body MRI 3 Tesla MR scanner (Achieva; Philips Healthcare, Best, the Netherlands) and a standard 8-channel head coil. For quality control, a spectroscopy phantom (i.e. the Philips Sphere A) was scanned every day before each MRS session to ensure magnetic field intensity stability. The phantom was a roundshape with a $10 \mathrm{~cm}$ diameter sealed ball. A single-voxel point-resolved spectroscopy (PRESS) pulse sequence was used time repetition [TR]/time echo [TE] $=2000 \mathrm{~ms} / 144 \mathrm{~ms}$, number of signal averages $[\mathrm{NSA}]=128$, voxel size $=20 \times 20 \times 20$ $\mathrm{mm}$, placed in the center of phantom) and dispersion of ratio was computed. Central magnetic field homogeneity and stability over time were demonstrated.

During a scan, participants were positioned supine and were instructed to lie comfortably and to remain stable. Foam pads and forehead-restraining straps were utilized to hold the head's position within the head coil. Following the acquisition of low-resolution localizer MR images, 3-plane 2D T2-weighted turbo spin echo images were acquired to facilitate identification of the anatomical landmarks for accurate positioning of the volume of interest (VOI). Smart Scan procedure (manufacturer supplied) was used to ensure accurate positioning of each patient for consistent anatomical landmarks across different scan sessions. Spectral data were acquired from three targeted VOIs (Figure 1), positioned within the bilateral TH, ACC, and the primary S1's posterior part of the paracentral gyrus (PPG; shown as the area within the green-line rectangle in Figure 1). B0 shimming (shown as the grid in red in Figure 1) was performed using the manufacturer-supplied phase map method with additional interactive manual shimming for full-width at half-maximum (FWHM) of $\leq 12 \mathrm{~Hz}$ with the unsuppressed water signal line width.

Data acquisition used 2D multiple voxel PRESS sequence $\left(\mathrm{TR} / \mathrm{TE}=2000 / 144 \mathrm{~ms}, \mathrm{NSA}=1\right.$, flip angle $=90^{\circ}$,
FOV $=16 \times 16 \mathrm{~mm}$, matrix $=16 \times 16$, voxel size $=1 \times 1 \times 1.5 \mathrm{~mm}$ ) lasting for 8' 26 " for each VOI. The spectra of the adjunct 4 voxels (i.e. $4 \times 4 \times 1.5 \mathrm{~mm}^{3}$ ) were averaged to produce the data for further analysis, resulting in four sets of spectrum data from four brain regions (Figure 1, yellow squares) located respectively in the left and right thalamus (THleft, TH-right), the ACC, and the posterior portion of the PPG in S1.

Spectral data analyses were performed separately for each VOI, using curve-fitting automation software (manufacturer supplied), which consisted of zero filling, Fourier transformation, baseline, and eddy current corrections. Levels of the metabolites of interest were normalized referencing the water-yielding quantification. The peak areas under the curve fitting of the NAA (2.02 ppm), Cr (3.02 ppm), and Cho $(3.20 \mathrm{ppm})$ were calculated for further analysis. Other peaks of the metabolites were not used in the analysis, as they do not demonstrate a sufficiently high signal-to-noise ratio using the MR spectroscopy sequence to support reliable quantification. Results are presented as ratios of NAA and Cho to $\mathrm{Cr}$ (i.e. NAA/Cr and $\mathrm{Cho} / \mathrm{Cr}$ ), using $\mathrm{Cr}$ as the internal reference as with common practice. ${ }^{26}$ Given the dispute of using $\mathrm{Cr}$ as reference, we also calculated Cho/NAA to further verify the result. ${ }^{27}$

\section{Statistics}

Group mean differences in the clinical measurements were examined using analysis of variance (ANOVA). Differences in the levels of metabolites and pain scores pre- and post- the intervention were examined using paired $t$-tests, separately for each subject group. Relationships between age, the pain scores, and the metabolites were tested using the Pearson correlation coefficients with $95 \%$ confidence intervals (CIs) and fitted using a linear regression model. In the regression analysis, outlier observations in the dataset with a leverage value greater than $2 p / n$, where $p$ represents the number of independent and $n$ is the total number of observations, were discarded from the model fitting. ${ }^{28}$ (One outlier value pair of a 47-year-old female MwoA patient was identified for the regression linking VAS and NAA/ $\mathrm{Cr}$ in TH-right, and none in TH-left or for any other conditions.) A multivariable linear model was used to examine the effect of acupuncture treatment using the correlation coefficient $B$ with $95 \% \mathrm{CI}$, adjusting for age, sex, headache history, and brain region for each subject group. To test the clinical importance of the analysis outcome, effect sizes (ESs) were calculated for the tests using Cohen's power 

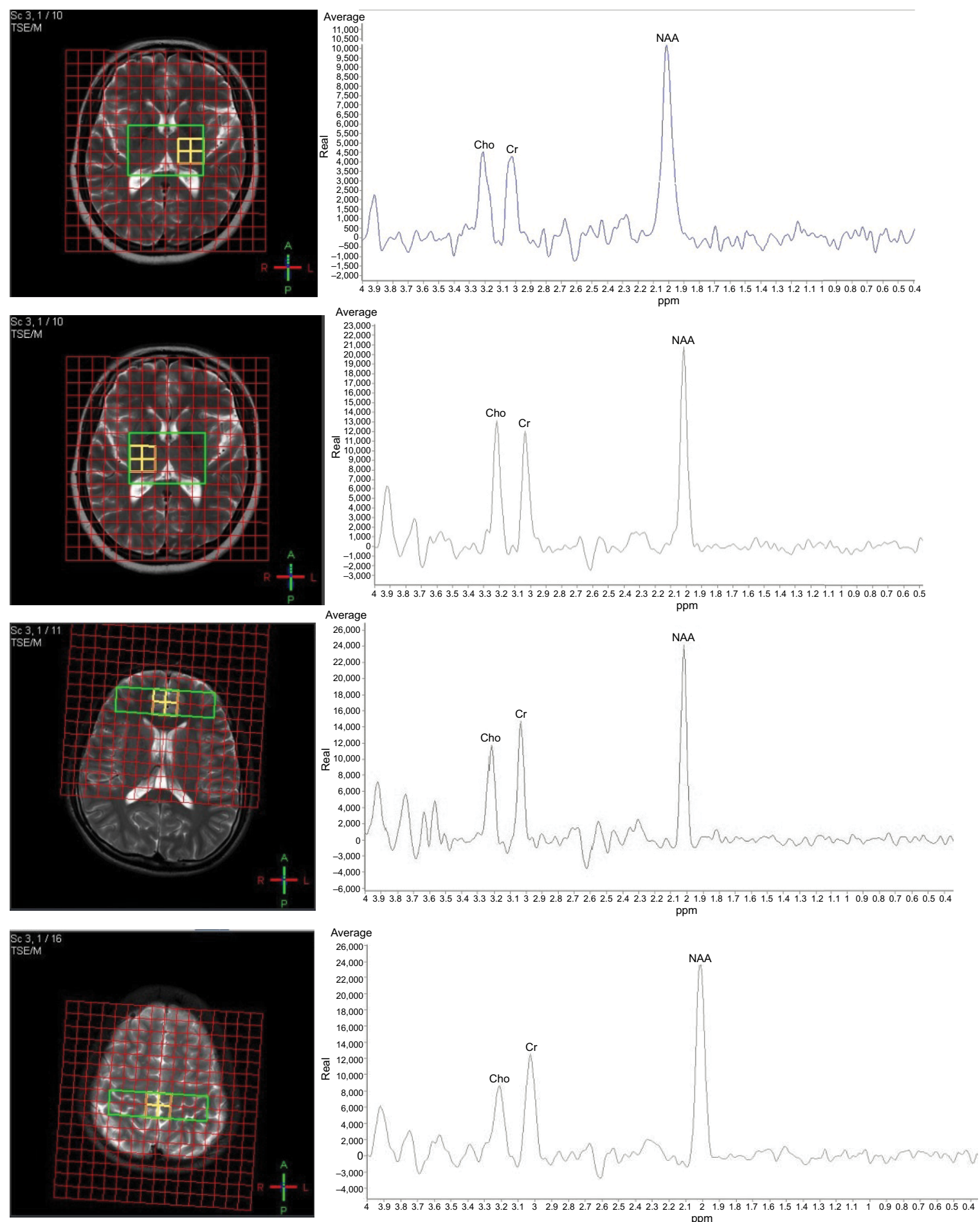

Figure I Locations of the targeted VOI superimposed on T2-weighted imaging (left panels) and the corresponding sample MRS spectra (right panels).

Note: From top to bottom: left thalamus (TH-left); right thalamus (TH-right); anterior cingulate gyrus (ACC); posterior part of paracentral gyrus (PPG). Red grids show B0 shimming; green-lined rectangles show the VOls; yellow squares show the voxels from which the spectra were retrieved and averaged for analysis.

Abbreviations: VOls, voxels of interest; MRS, magnetic resonance spectroscopy; NAA: N-acetylaspartate; Cr: creatine; Cho: choline-containing compounds.

analysis. ${ }^{29,30}$ All the analyses were carried out using SPSS v21.0 (IBM Corp, Armonk, NY, USA) and codes developed using Matlab v2017 (MathWorks Inc, Natick, MA, USA). The statistical significance level was set at $p<0.050$.

\section{Ethics}

This study protocol was approved by the Ethics Committee of the Beijing Hospital. A written informed consent was obtained from each participant. Additional ethical approval 
for secondary data analysis was obtained from Fraser Health Research Ethics Board.

\section{Results}

Out of the 45 participants, one male HC subject dropped out, resulting in $14 \mathrm{HC}, 15 \mathrm{MwoA}$, and $15 \mathrm{CH}$ for statistical analysis. Characteristics of the participants showed significant differences in the mean age, headache history, headache intensity (as measured using the VAS), and duration of headache attacks among the subject groups $(p<0.050)$. The two patient groups had similar headache frequencies ( $p>0.050$; Table 2).

Acupuncture treatment resulted in a significant reduction in the mean VAS score in MwoA patients ( $7.0 \pm 1.5 \mathrm{vs}$. $6.3 \pm 1.3, \mathrm{~T}=-6.20, \mathrm{ES}=0.50, p<0.001$; Table 2 ), demonstrating an improvement in clinical headache outcome. A small reduction in the mean duration of headache attacks was also observed in MwoA (12.1 \pm 10.5 vs. 10.9 $\pm 10.1, \mathrm{~T}=-2.60$, $\mathrm{ES}=0.12, p=0.023$; Table 2). These were in contrast to a lack of pre- vs. post-acupuncture difference in the $\mathrm{CH}$ patients $(p>0.050)$.

Acupuncture treatment was associated with a significantly increased mean level of $\mathrm{NAA} / \mathrm{Cr}$ in the TH (i.e. TH-left and TH-right) in MwoA patients; a medium ES was found in TH-

Table 2 Characteristics of the sample

\begin{tabular}{|c|c|c|c|}
\hline Group & $\begin{array}{l}\text { Migraine } \\
\text { without aura }\end{array}$ & $\begin{array}{l}\text { Cervicogenic } \\
\text { headache }\end{array}$ & $\begin{array}{l}\text { Healthy } \\
\text { control }\end{array}$ \\
\hline $\mathrm{N}$ (female/male) & $10 / 5$ & $10 / 5$ & $10 / 4$ \\
\hline Age (years) & $\begin{array}{l}34.8 \pm 10.6 \\
{[18-55]}\end{array}$ & $\begin{array}{l}43.3 \pm 12.1 \\
{[20-60]}\end{array}$ & $\begin{array}{l}22.4 \pm 1.1 \\
{[20-24]}\end{array}$ \\
\hline Headache history (years) & $\begin{array}{l}16.3 \pm 9.8 \\
{[2-40]}\end{array}$ & $\begin{array}{l}4.3 \pm 3.0 \\
{[2-10]}\end{array}$ & 0 \\
\hline \multicolumn{4}{|c|}{ Baseline clinical outcomes } \\
\hline Headache intensity $\left(\mathrm{VAS}^{\wedge}\right)$ & $\begin{array}{l}7.0 \pm 1.5^{*} \\
{[4-8]}\end{array}$ & $\begin{array}{l}4.7 \pm 1.0 \\
{[3-6]}\end{array}$ & 0 \\
\hline $\begin{array}{l}\text { Headache frequency } \\
\text { (times/month) }\end{array}$ & $\begin{array}{l}4.0 \pm 3.4 \\
{[1-12]}\end{array}$ & $\begin{array}{l}3.9 \pm 3.5 \\
{[1-15]}\end{array}$ & NA \\
\hline $\begin{array}{l}\text { Duration of headache }{ }^{\#} \\
\text { (hours/per time) }\end{array}$ & $\begin{array}{l}12.1 \pm 10.5^{*} \\
{[1-24]}\end{array}$ & $\begin{array}{l}3.5 \pm 3.0 \\
{[1-12]}\end{array}$ & NA \\
\hline \multicolumn{4}{|c|}{ Follow-up clinical outcomes } \\
\hline Headache intensity $\left(\mathrm{VAS}^{\wedge}\right)$ & $\begin{array}{l}6.3 \pm 1.3^{*} \\
{[4-8]}\end{array}$ & $\begin{array}{l}4.5 \pm 0.8 \\
{[3-6]}\end{array}$ & 0 \\
\hline $\begin{array}{l}\text { Headache frequency } \\
\text { (times/month) }\end{array}$ & $\begin{array}{l}3.6 \pm 2.7 \\
{[1-10]}\end{array}$ & $\begin{array}{l}3.9 \pm 3.4 \\
{[1-15]}\end{array}$ & NA \\
\hline $\begin{array}{l}\text { Duration of headache } e^{\#} \\
\text { (hours/per time) }\end{array}$ & $\begin{array}{l}10.9 \pm 10.1 * \\
{[1-24]}\end{array}$ & $\begin{array}{l}3.3 \pm 2.8 \\
{[1-12]}\end{array}$ & NA \\
\hline
\end{tabular}

Notes: Data are presented as mean \pm standard deviation; value ranges are given in the square brackets. ^VAS: visual analog scores $\left(0=\right.$ no pain to $10=$ worst pain ever) ${ }^{\S}$ Defined as the number of headaches separated by pain-free intervals of at least 48 hours within the past 4 weeks. "Defined as the time in hours from the beginning until the end of the headache episode. *Significant difference between baseline and follow-up.

Abbreviations: VAS, visual analog score; NA, not applicable. left $(1.90 \pm 0.22$ vs. $2.11 \pm 0.35, \mathrm{~T}=3.43, \mathrm{ES}=0.68, p=0.006)$ and a large $\mathrm{ES}$ in TH-right $(1.83 \pm 0.18$ vs. $1.96 \pm 0.14, \mathrm{~T}=3.38$, $\mathrm{ES}=0.81, p=0.006)$. No difference was seen in the $\mathrm{CH}$ or $\mathrm{HC}$ between pre- and post-acupuncture (Table 3; Figure 2A).

In the ACC region, varied responses were observed in NAA/Cr for different groups: no change in MwoA, a decrease in $\mathrm{CH}$ (and an increase in Cho/NAA) and an increase in $\mathrm{HC}$ (Table 3; Figure 2A). In the PPG region, a change in NAA/Cr was not observed for any group ( $p>0.05$; Table 3; Figure 2A). No change in $\mathrm{Cho} / \mathrm{Cr}$ was observed, regardless of group or location ( $p>0.05$; Table 3; Figure 2B).

A significant correlation between NAA/Cr and VAS was observed for all participants ( $n=44$; dashed lines in Figure 3) in TH-left and TH-right both at baseline (i.e. pretreatment) and post-treatment follow-up (TH-left baseline $\mathrm{r}=-0.60$, $p<0.001$, Figure 3, left upper panel; TH-left follow-up $\mathrm{r}=-0.45, p=0.002$, Figure 3, left lower panel; TH-right baseline $\mathrm{r}=-0.66, p<0.001$, Figure 3, right upper panel; TH-right follow-up $r=-0.37, p=0.013$, Figure 3, right lower panel).

A closer relationship with medium to large EFs between $\mathrm{NAA} / \mathrm{Cr}$ and VAS was found for individuals with MwoA (solid lines in Figure 3) in the thalami region at both time points (TH-left baseline $\mathrm{r}=-0.70, p=0.004, \mathrm{n}=15$, Figure 3, left upper panel; TH-left follow-up $\mathrm{r}=-0.71, p=0.003, \mathrm{n}=15$, Figure 3, left lower panel; TH-right baseline $\mathrm{r}=-0.64, p=0.014$, $\mathrm{n}=14$, Figure 3, right upper panel; TH-right follow-up $\mathrm{r}=-0.66$, $p=0.010, \mathrm{n}=14$, Figure 3 , right lower panel). No such correlation was found for $\mathrm{CH}$ or $\mathrm{HC}\left(\mathrm{r}^{\prime} \mathrm{s}<0.15, p\right.$ 's $\left.>0.584\right)$.

Multivariable analyses examining the effect of treatment showed consistent age and brain region differences in NAA/ $\mathrm{Cr}$ in each subject group (B correlation coefficient $=-0.02$, $95 \% \mathrm{CI}=-0.04$ to $-0.01, p=0.002$ in $\mathrm{MwoA} ; \mathrm{B}=-0.01, \mathrm{CI}=-$ 0.02 to $-0.00, p=0.001$ in $\mathrm{CH} ; \mathrm{B}=-0.16, \mathrm{CI}=-0.24$ to -0.09 , $p<0.001$ in HC; Table 4). However, a significant treatment effect was only found in MwoA ( $\mathrm{B}=0.16, \mathrm{CI}=0.06$ to 0.26 . $p=0.002$; Table 4) and not in other groups, confirming a brain metabolic response to acupuncture treatment of migraine.

\section{Discussion}

In this study, we investigated brain metabolite changes in response to acupuncture treatment in a migraine patient sample. We also examined the relationships between the metabolite changes and clinical outcomes. The results showed a significant increase of the NAA/Cr post-acupuncture treatment with a large effect size, in brain regions important for transmitting sensation of pain, e.g. the thalami. In addition, the level of NAA/Cr was correlated with the reduction of headache intensity. This study represents the first MRSI work 
Table 3 Levels of metabolites comparing different subject groups at pre- and post-intervention conditions

\begin{tabular}{|c|c|c|c|c|c|}
\hline $\begin{array}{l}\text { Brain } \\
\text { region }\end{array}$ & $\begin{array}{l}\text { Metabolite } \\
\text { ratio }\end{array}$ & Time & $\begin{array}{l}\text { Migraine without aura } \\
(n=15)\end{array}$ & $\begin{array}{l}\text { Cervicogenic headache } \\
(n=15)\end{array}$ & $\begin{array}{l}\text { Healthy control } \\
(n=14)\end{array}$ \\
\hline \multirow[t]{9}{*}{ Left thalamus } & $\mathrm{NAA} / \mathrm{Cr}$ & 1 & $1.91 \pm 0.22$ & $1.94 \pm 0.27$ & $2.36 \pm 0.36$ \\
\hline & & 2 & $2.11 \pm 0.35$ & $1.86 \pm 0.20$ & $2.35 \pm 0.41$ \\
\hline & Statistics $\mathrm{T}(\mathrm{P})$ & & $3.43(0.006)^{*}$ & $-1.49(0.160)$ & $-0.11(0.913)$ \\
\hline & $\mathrm{Cho} / \mathrm{Cr}$ & 1 & $1.00 \pm 0.08$ & $1.08 \pm 0.09$ & $0.95 \pm 0.16$ \\
\hline & & 2 & $1.05 \pm 0.13$ & $1.01 \pm 0.06$ & $1.04 \pm 0.13$ \\
\hline & Statistics $T(P)$ & & $1.63(0.132)$ & $-2.11(0.068)$ & $1.69(0.116)$ \\
\hline & Cho/NAA & 1 & $0.52 \pm 0.08$ & $0.58 \pm 0.09$ & $0.4 I \pm 0.08$ \\
\hline & & 2 & $0.51 \pm 0.10$ & $0.57 \pm 0.08$ & $0.45 \pm 0.08$ \\
\hline & Statistics $\mathrm{T}(\mathrm{P})$ & & $-1.00(0.340)$ & $-0.73(0.488)$ & $1.96(0.072)$ \\
\hline \multirow[t]{9}{*}{ Right thalamus } & $\mathrm{NAA} / \mathrm{Cr}$ & 1 & $1.83 \pm 0.18$ & $1.82 \pm 0.18$ & $2.19 \pm 0.20$ \\
\hline & & 2 & $1.96 \pm 0.14$ & $1.78 \pm 0.15$ & $2 .|2 \pm 0.3|$ \\
\hline & Statistics $\mathrm{T}(\mathrm{P})$ & & $3.38(0.006)^{*}$ & $-1.18(0.256)$ & $-0.88(0.395)$ \\
\hline & Cho/Cr & I & $1.00 \pm 0.07$ & $1.08 \pm 0.06$ & $0.98 \pm 0.14$ \\
\hline & & 2 & $1.04 \pm 0.10$ & $1.04 \pm 0.07$ & $1.01 \pm 0.09$ \\
\hline & Statistics $\mathrm{T}(\mathrm{P})$ & & $1.59(0.140)$ & $-1.83(0.104)$ & $0.70(0.497)$ \\
\hline & Cho/NAA & I & $0.55 \pm 0.06$ & $0.60 \pm 0.07$ & $0.45 \pm 0.06$ \\
\hline & & 2 & $0.53 \pm 0.06$ & $0.59 \pm 0.06$ & $0.48 \pm 0.05$ \\
\hline & Statistics $T(P)$ & & $-1.06(0.311)$ & $-1.23(0.292)$ & I.84 (0.089) \\
\hline \multirow[t]{9}{*}{ Anterior cingulate cortex } & $\mathrm{NAA} / \mathrm{Cr}$ & I & $1.5 \mathrm{I} \pm 0.15$ & $I .5 \mathrm{I} \pm 0.1 \mathrm{I}$ & $1.55 \pm 0.15$ \\
\hline & & 2 & $1.5 I \pm 0.13$ & $1.4 \mathrm{I} \pm 0.13$ & $1.66 \pm 0.09$ \\
\hline & Statistics $\mathrm{T}(\mathrm{P})$ & & $-0.12(0.904)$ & $-3.92(0.002)^{*}$ & $2.83(0.014)^{*}$ \\
\hline & Cho/Cr & 1 & $1.00 \pm 0.14$ & $1.04 \pm 0.06$ & $0.97 \pm 0.08$ \\
\hline & & 2 & $1.05 \pm 0.13$ & $1.06 \pm 0.08$ & $0.95 \pm 0.08$ \\
\hline & Statistics $\mathrm{T}(\mathrm{P})$ & & $1.48(0.168)$ & $0.79(0.454)$ & $-0.59(0.565)$ \\
\hline & Cho/NAA & 1 & $0.67 \pm 0.12$ & $0.69 \pm 0.05$ & $0.62 \pm 0.09$ \\
\hline & & 2 & $0.70 \pm 0.13$ & $0.76 \pm 0.07$ & $0.57 \pm 0.05$ \\
\hline & Statistics $\mathrm{T}(\mathrm{P})$ & & $1.32(0.215)$ & $2.86(0.021)^{*}$ & $-2.15(0.05 \mathrm{I})$ \\
\hline Posterior part of & $\mathrm{NAA} / \mathrm{Cr}$ & 1 & $1.62 \pm 0.11$ & $1.62 \pm 0.13$ & $1.73 \pm 0.08$ \\
\hline \multirow[t]{8}{*}{ paracentral gyrus } & & 2 & $1.67 \pm 0.14$ & $1.54 \pm 0.19$ & $1.71 \pm 0.15$ \\
\hline & Statistics $T(P)$ & & $1.64(0.130)$ & $-2.11(0.067)$ & $-0.53(0.607)$ \\
\hline & $\mathrm{Cho} / \mathrm{Cr}$ & 1 & $0.76 \pm 0.06$ & $0.8 \mathrm{I} \pm 0.03$ & $0.76 \pm 0.06$ \\
\hline & & 2 & $0.79 \pm 0.07$ & $0.78 \pm 0.06$ & $0.78 \pm 0.08$ \\
\hline & Statistics $T(P)$ & & $1.14(0.280)$ & $-1.51(0.17 \mid)$ & $0.57(0.577)$ \\
\hline & Cho/NAA & 1 & $0.50 \pm 0.08$ & $0.50 \pm 0.03$ & $0.44 \pm 0.03$ \\
\hline & & 2 & $0.47 \pm 0.04$ & $0.5 \mathrm{I} \pm 0.08$ & $0.45 \pm 0.04$ \\
\hline & Statistics $T(P)$ & & $-0.92(0.380)$ & $0.43(0.676)$ & $1.43(0.176)$ \\
\hline
\end{tabular}

Note: I=pre-intervention scan; $2=$ post-intervention scan; ${ }^{*} p<0.05$.

Abbreviations: NAA, N-acetylaspartate; $\mathrm{Cr}$, creatine; Cho, choline-containing compounds; T, $t$-test; $\mathrm{P}, p$-value.

to investigate the effect of acupuncture treatment on brain metabolites in migraine, evidencing a positive response of brain metabolites to acupuncture therapy. The data contributed to the literature with new information in understanding the mechanism of acupuncture in treating migraine.
Multiple medications have been proven to be particularly efficacious and they play the most important role in the management of migraine. ${ }^{2,3}$ Meanwhile, given the complexity of the migraine disorder and that not all patients demonstrate highly satisfactory responsiveness, ${ }^{31}$ a number of non- 
A

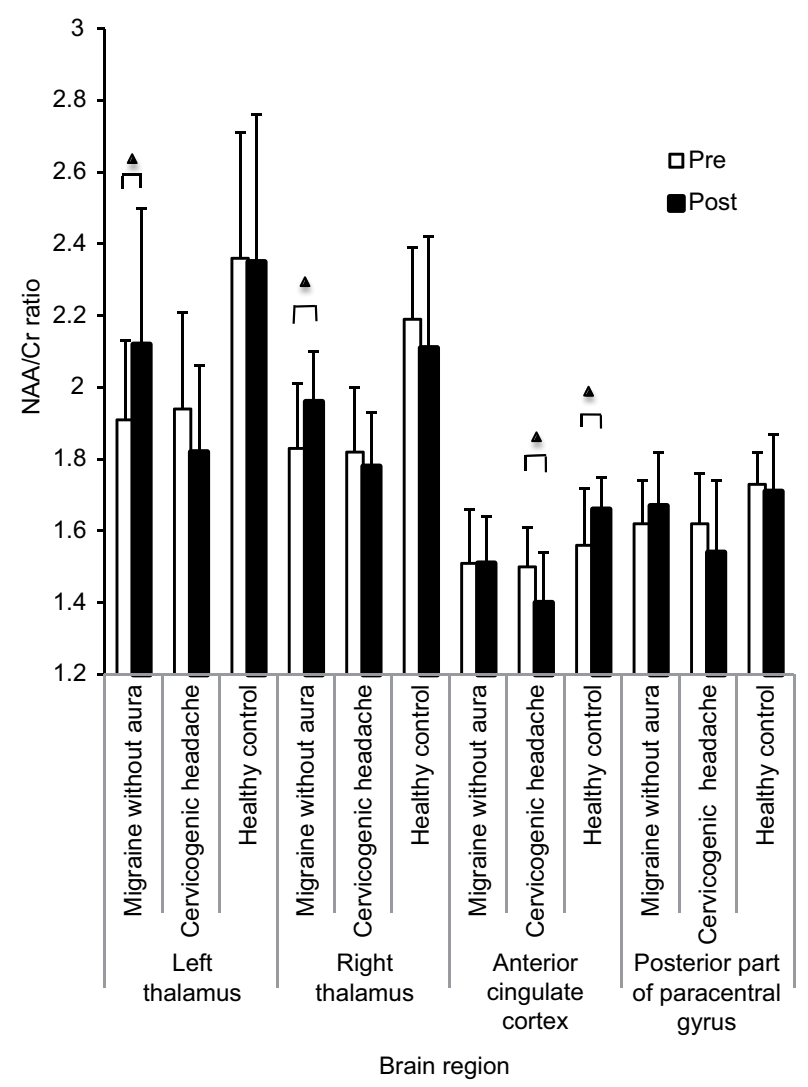

B

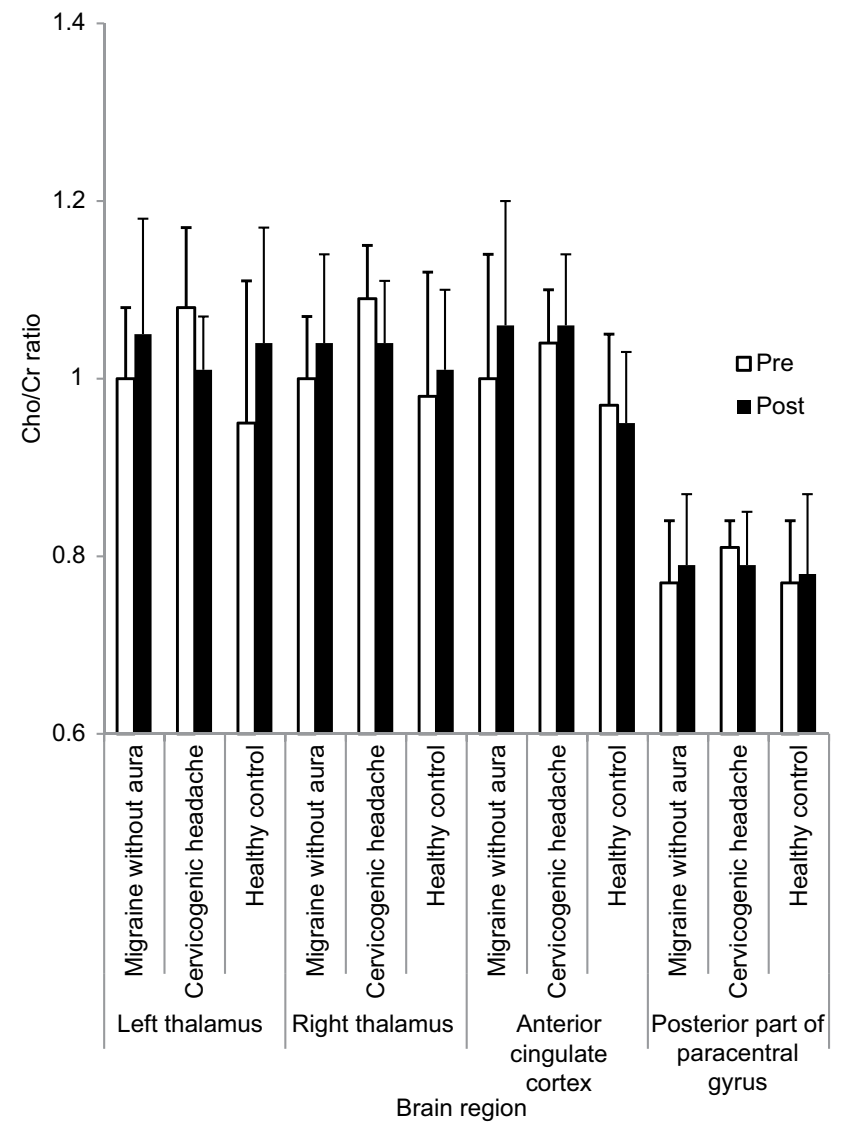

Figure 2 Comparison of the level of metabolite ratios between subject groups at pre-and post-intervention conditions for (A) NAA/Cr and (B) Cho/Cr.

Notes: $\boldsymbol{\Delta}=$ Significant difference at $p<0.050$ between pre- and post-intervention conditions. For each test, $\mathrm{n}=15$ for each of the $\mathrm{MwoA}$ and $\mathrm{CH}$ patient groups, and $\mathrm{n}=14$ for the HC group.

Abbreviations: NAA, N-acetylaspartate; $\mathrm{Cr}$, creatine; $\mathrm{Cho}$, choline-containing compounds; MwoA, migraine without aura; $\mathrm{CH}$, cervicogenic headache; HC, healthy control.

pharmaceutical approaches with proven clinical benefits have been approved as alternative therapies. ${ }^{4}$ Previous studies have demonstrated the treatment effect of acupuncture on migraine. ${ }^{32,33}$ For example, Wang et al conducted a clinical trial and showed that verum acupuncture relieved the pain and reduced the usage of acute medication. ${ }^{32} \mathrm{Li}$ et al proposed that valid acupuncture was associated with long-term reduction of migraine recurrence compared with sham acupuncture. ${ }^{33}$ It has been suggested that the effect of acupuncture treatment involves the nociceptive modulation that is associated with several pathological processes including spread activation and deactivation of cortical trigeminovascular pain pathways. ${ }^{34-36}$ Previous work has also suggested the role of NAA deficits in migraine. ${ }^{37}$ As shown in our previous work, there is a decrease in the level of NAA in the thalamus in MwoA patients. ${ }^{13}$ Even so, until now, whether a brain metabolite change may have a role in acupuncture treatment has not been understood. Here, our data showed that indeed, an increase of NAA/Cr (while Cr remained relatively stable) in bilateral TH was the result of acupuncture therapy, associated with a relief of clinical pain. Generally recognized as a marker of normal neuronal activity, synthesized/located in neuronal mitochondria, NAA decrease indicates mitochondrial dysfunction. ${ }^{38}$ Thus, an increase of NAA may reflect a recovery of neuronal activity of the thalamus through mediating nociceptive modulation. ${ }^{39}$

Our data must be interpreted with caution. First, our study involved a relatively small group of patient participants, a single study site and one experienced practitioner for acupuncture treatment. Although all the data points were included in the analyses (except for one outlier in regressing NAA/Cr and VAS in TH-right for MwoA) the small sample size can limit the generalizability. Further studies with larger samples size will be needed to better generalize the research finding.

Second, due to difficulties with recruitment, most of our MwoA and $\mathrm{CH}$ patients were mid-aged adults, while those of the $\mathrm{HC}$ group were younger adults. Previous research has suggested an inverse relationship between NAA and age. ${ }^{40}$ Our data showed a comparatively higher level of NAA/Cr in the $\mathrm{HC}$ subjects than in the patients, likely related to the mean age difference. Due to this reason, we restricted the analyses 

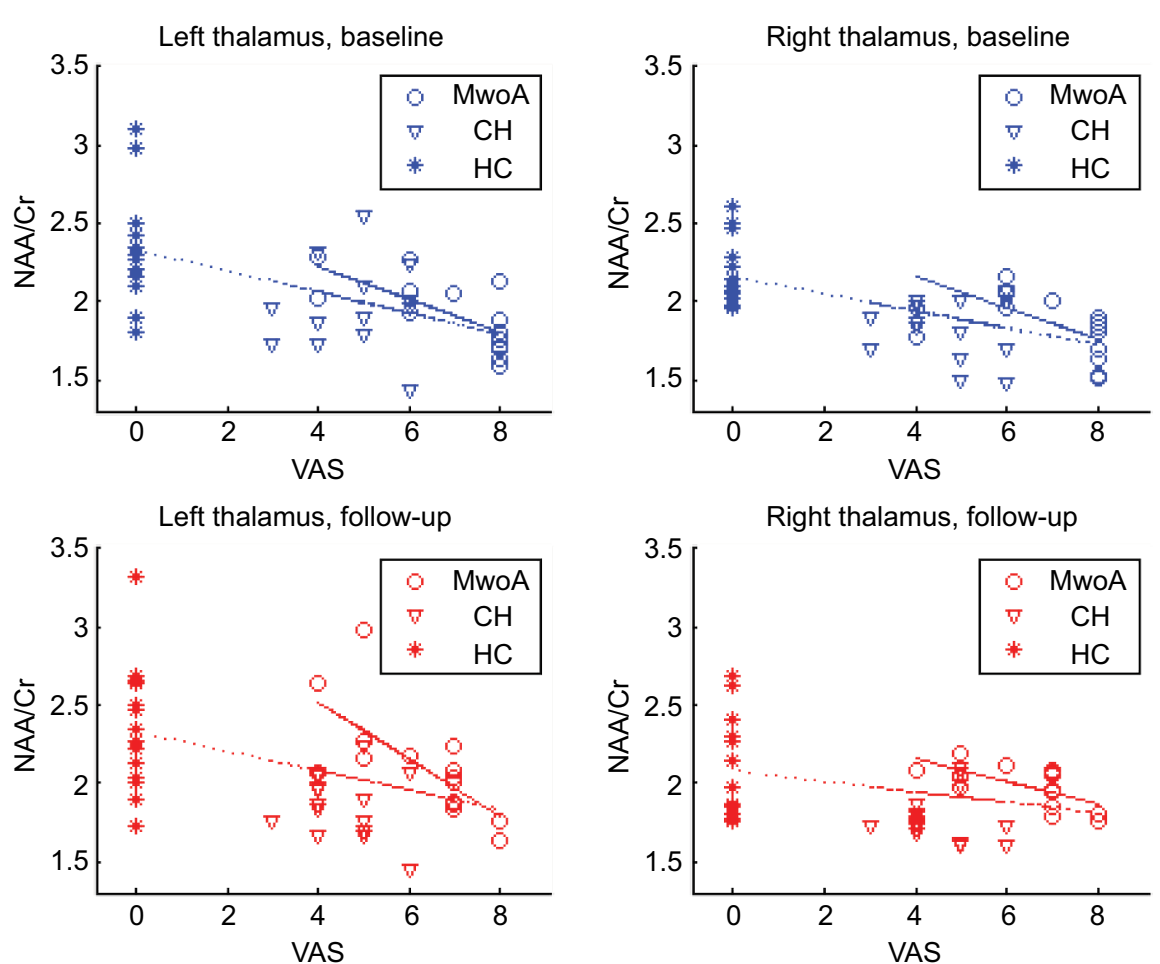

Figure 3 The relationship between NAA/Cr and VAS in thalamus at baseline and follow-up conditions.

Notes: Symbols represent the observational data and lines represent the linear fitting $f(x)=a+b x$, where $x$ is the VAS scores and $y$ is the level of metabolites. Solid lines represent the regressions for MowA patients ( $\mathrm{al}, \mathrm{bI})$, and dashed lines for the entire sample $(a 2, b 2)$. Left top panel: $\mathrm{al}=2.65, \mathrm{bl}=-0 . \mathrm{II}(\mathrm{n}=\mathrm{I} \mathrm{I}, \mathrm{r}=-0.70, \mathrm{p}=0.004)$; $\mathrm{a} 2=2.33$, $b 2=-0.07(n=44, r=-0.60, p<0.00 I)$. Left bottom panel: $a l=3.24, b I=-0.18(n=15, r=-0.7 I, p=0.003) ; a 2=2.32, b 2=0.06(n=44, r=-0.45, p=0.002)$. Right top panel: al=2.55, $\mathrm{bl}=-0.10(\mathrm{n}=14, \mathrm{r}=-0.64, p=0.0 \mathrm{I}) ; \mathrm{a} 2=2.16, \mathrm{~b} 2=-0.05(\mathrm{n}=44, \mathrm{r}=-0.66, p<0.00 \mathrm{I})$. Right bottom panel: $\mathrm{l} \mathrm{I}=2.44, \mathrm{bI}=-0.07(\mathrm{n}=\mathrm{I4}, \mathrm{r}=-0.66, p=0.0 \mathrm{I} 0) ; \mathrm{a}=2.07, \mathrm{~b} 2=-0.0(\mathrm{n}=44$, $r=-0.37, p=0.013)$.

Abbreviations: NAA, N-acetylaspartate; $\mathrm{Cr}$, creatine; MwoA, migraine without aura; $\mathrm{CH}$, cervicogenic headache; $\mathrm{HC}$, healthy control; VAS, visual analog scale.

to comparing the measurements pre- and post-treatment in the same individuals rather than across groups. We have also identified a negative association between age and NAA/Cr at both baseline and follow-up in both patient groups. However, the pre- vs. post-treatment change score was not associated with age $(\mathrm{r}=-0.02, p=0.869)$, while the association between age and the clinical pain scores was significant at neither baseline nor follow-up. Moreover, multivariable analyses examining the effect of treatment adjusting age in each group showed a clear age impact on acupuncture efficacy in all the three subject groups. However, only in the MwoA group there was also a significant treatment effect (Table 4),

Table 4 Effect of acupuncture treatment on the level of NAA/Cr adjusting for age, sex, brain region, and headache history for each subject group

\begin{tabular}{llllllll}
\hline Group & Co-variables & B & Std. error & $\mathbf{9 5 \%}$ Cl & Beta & T & P-value \\
\hline Migraine without & Treatment (post vs. pre) & 0.16 & 0.05 & $0.06-0.26$ & 0.33 & 3.28 & 0.002 \\
aura & Age (year) & -0.02 & 0.01 & $-0.04--0.01$ & -0.93 & -3.24 & 0.002 \\
& Sex (female vs. male) & -0.05 & 0.06 & $-0.16-0.06$ & -0.10 & -0.96 & 0.343 \\
& Location (left vs. right) & 0.11 & 0.05 & $0.01-0.21$ & 0.21 & 2.13 & 0.038 \\
Cervicogenic & Headache history (year) & 0.01 & 0.01 & $-0.00-0.02$ & 0.42 & 1.48 & 0.144 \\
headache & Treatment (post vs. pre) & -0.05 & 0.05 & $-0.15-0.04$ & -0.13 & -1.15 & 0.255 \\
& Age (year) & -0.01 & 0.00 & $-0.02--0.00$ & -0.47 & -3.62 & 0.001 \\
& Sex (female vs. male) & -0.13 & 0.05 & $-0.23--0.02$ & -0.28 & -2.32 & 0.024 \\
Healthy control & Location (left vs. right) & 0.10 & 0.05 & $0.00-0.19$ & 0.23 & 2.08 & 0.042 \\
& Headache history (year) & -0.01 & 0.01 & $-0.03-0.02$ & -0.06 & -0.51 & 0.615 \\
& Treatment (post vs. pre) & -0.04 & 0.07 & $-0.19-0.10$ & -0.06 & -0.59 & 0.559 \\
& Age (year) & -0.16 & 0.04 & $-0.24--0.09$ & -0.52 & -4.37 & 0.000 \\
& Sex (female vs. male) & -0.06 & 0.09 & $-0.24-0.11$ & -0.08 & -0.72 & 0.478 \\
& Location (left vs. right) & 0.20 & 0.07 & $0.06-0.35$ & 0.30 & 2.77 & 0.008 \\
\hline
\end{tabular}

Note: B represents the correlation coefficient.

Abbreviation: NA, not applicable. 
suggesting that the differences in brain metabolic activity in MwoA reflected a valid treatment effect.

In addition, quantification of metabolites was focused on the dominant peak representing each chemical of interest. It is known that with certain metabolites, quantification may also involve smaller peaks. For example, the most prominent peak of NAA is at $2.01 \mathrm{ppm}$, whereas a few smaller NAA peaks can overlap with glutamate and certain macromolecules. On the other hand, our analysis is to compare the same individuals over time. Given the overlap and relative low signal-to-noise ratio with some smaller peaks, focusing on the dominant peak can ensure effective analysis without introducing less reliable peaks. Previous studies have suggested a correlation between different peaks for calculating metabolite concentrations. ${ }^{27}$

Furthermore, our study was only focused on identifying a possible change of brain metabolites in response to acupuncture (and its possible associations with clinical pain scores) in MwoA but not $\mathrm{CH}$. Even though including the two reference groups has led to the differentiation of a responsive neurometabolite change in MwoA from $\mathrm{CH}$ (using non-targeting acupoints) or $\mathrm{HC}$ (using a sham acupuncture), the HC group with sham acupuncture may have only controlled for pain conduction due to acupuncture-induced stimulation regardless of sham acupoints. ${ }^{24}$ Alternatively, a true acupuncture with HC subjects could serve as a useful sham condition, even though it could be less favorable ethically to administer acupuncture treatment for people who do not have a symptom. ${ }^{41}$ In addition, previous studies have reported effective acupuncture treatment on $\mathrm{CH}^{21,22}$ In our study, the treatment effect on $\mathrm{CH}$ was not as effective as that on MwoA, as we used acupuncture points for targeting MwoA that are different from the primary acupoints for targeting $\mathrm{CH} .{ }^{21,22}$ Further studies using valid primary acupoints for treating $\mathrm{CH}$ can help us to better understand the neurometabolic responses of $\mathrm{CH}$ to acupuncture.

In spite of these limitations, the study demonstrates several strengths. The spectroscopy data were studied in multiple brain regions, each with known importance for pain transmission of specific functions. ${ }^{26}$ The neural pathways for afferent nociception involves trigeminal nerve fibers (firstorder neurons), spinal trigeminal nucleus (second-order neurons), and the thalamus (third-order neurons), so that alterations of the thalamic modulation of nociceptive input may contribute to pain perception during a headache attack, whereas a thalamic NAA increase can be an indication of recovery of the modulation. ${ }^{26}$ The $\mathrm{ACC}$, on the other hand, is involved in the emotional response to pain (affective aspects of pain perception), as well as in a behavior and reinforcement response to pain. ${ }^{42}$ This provides an explanation why in $\mathrm{CH}$, the acupuncture procedure did not benefit thalamic modulation but was associated with a decrease of NAA/Cr in the ACC. This emphasizes the importance of applying the treatment on the valid acupoints for targeted syndromes or conditions based on TCM. ${ }^{19}$ By the same token, a change of the metabolites was not found in the PPG in any group. This is not surprising because even though PPG is involved in encoding of the location, intensity and duration, it is not involved in the modulation of headache, as neurons in this region receive nociceptive signals from body and limbs, not head and face. ${ }^{43}$

The strength of the study also arises from the collection of both the metabolic and clinical data before and after the acupuncture treatment. These data support a possible correlation between brain metabolites of interest and clinical measurement scores and can help justify the clinical mindfulness of the imaging finding. The association of thalamic NAA increase with clinical pain relief in patients who received valid acupuncture demonstrated the treatment effect. The data also suggested that a sufficient level of the metabolites is necessary to ease headache symptoms.

With MR spectral data collection, subjects have previous experience with anatomical MRI scans so that a possible impact of first attempt effect can be minimized. With clinical data collection, the subject enrolment protocol has controlled for sex, medicine history, and other disease history which may influence brain metabolite changes. For the possible confounders that could not be controlled, their potential impacts were analyzed and adjusted using multivariate models.

In addition to examining the statistical significance of the results, we also evaluated the clinical importance of the findings. In respect to brain metabolites, the effect sizes between pre-and post-treatment for MwoA ranged from medium to large, while the ESs were relatively small for the clinical pain scores, likely indicating a greater sensitivity of the detection of brain metabolite changes.

\section{Conclusion}

Migraine is a common headache disorder causing significant healthcare and socioeconomic concerns. Acupuncture has been proven to be effective as an alternative therapy to aid in medication treatment in helping patients with the disorder, but its widespread application has not been fully realized due to lack of understanding of the underlying mechanism 
on the nervous system. The present study provided the first evidence demonstrating changes of brain metabolites in response to acupuncture therapy in migraine in relation to clinical outcomes. The study has shown an example of using MR spectroscopy technology in understanding the clinical importance of acupuncture in treating migraine.

\section{Clinical implications}

- The study showed evidence validating acupuncture therapy in treating migraine.

- It provided novel information on brain metabolic response to acupuncture therapy.

- It suggested the possible involvement of a recovery of neuronal mitochondrial function of the pain pathway in migraine treatment.

\section{Acknowledgments}

This study was supported by grants from the National Natural Science Foundation of China (30901928), Capital's Funds for Health Improvement and Research (2014-4-4052), and the Surrey Hospitals and Outpatient Centre Foundation (G2017-001). The authors acknowledge Dr Ping Zeng for consultation on statistics and Ms B Chinda for proofreading of the manuscript.

\section{Author contributions}

Experimental design and paper structure (TG, LL, YJ, MC, XS); MRSI data acquisition and processing (TG, JC); clinical data collection (YJ); acupuncture treatment (LL); data analysis, result presentation and first draft (TG, XS); result interpretation and discussion (TG, LL, JC, YJ, RCND, MC, XS). All authors contributed towards data analysis, drafting and critically reviewing and revising the paper, and agree to be accountable for all aspects of the work.

\section{Disclosure}

TG received a Fellowship Award from Beijing Hospital to conduct postdoctoral research in Canada. The authors report no other conflicts of interest in this work.

\section{References}

1. Headache Classification Subcommittee of the International Headache Society. The international classification of headache disorders. Cephalalgia. 2004;24 (Suppl):9-160.

2. Taheraghdam AA, Amiri H, Shojaan H, Shamsvahdati S, Houshyar Y. Intravenous dexamethasone versus morphine in relieving of acute migraine headache. Pak J Biol Sci. 2011;14(12):682-687.

3. Javid N, Raieian-Kopaei M. Is dexamethasone a suitable alternative for dihydroergotamine on migraine attacks? Int J Pharmacol. 2005;1(4):357-359.
4. Schiapparelli P, Allais G, Castagnoli Gabellari I, et al. Non-pharmacological approach to migraine prophylaxis: part II. Neurol Sci. 2010;31 (Suppl 1):s137-s139.

5. Linde K, Allais G, Brinkhaus B, Manheimer E, Vickers A, White AR. Acupuncture for migraine prophylaxis. Cochrane Database Syst Rev. 2009;1:CD001218.

6. Linde K, Allais G, Brinkhaus B, et al. Acupuncture for the prevention of episodic migraine. Cochrane Database Syst Rev. 2016;6:CD001218.

7. $\mathrm{Fu} \mathrm{H}$. What is the material base of acupuncture? The nerves! Med Hypotheses. 2000;54:358-359.

8. Harris RE, Zubieta JK, Scott DJ, Napadow V, Gracely RH, Clauw DJ. Traditional Chinese acupuncture and placebo (sham) acupuncture are differentiated by their effects on u-opioid receptors (MORs). Neuroimage. 2009;47(3):1077-1085.

9. Deng QS, Fang ZC, Yin Y. Ionic mechanism of acupuncture on improvement of learning and memory in age mammals. Am J Chin Med. 1995;23(1):1-9.

10. Sánchez del Rio M, Alvarez Linera J. Functional neuroimaging of headaches. Lancet Neurol. 2004;3(11):645-651.

11. Noseda R, Jakubowski M, Kainz V, Borsook D, Burstein R. Cortical projections of functionally identified thalamic trigeminovascular neurons: implications for migraine headache and its associated symptoms. J Neurosci. 2011;31(40):14204-14217.

12. Coghill RC, Sang CN, Maisog JM, Iadorola MJ. Pain intensity processing within the human brain: a bilateral, distributed mechanism. $J$ Neurophysiol. 1999;82(4):1934-1943.

13. Gu T, Ma XX, Xu YH, Xiu JJ, Li CF. Metabolite concentration ratios in thalami of patients with migraine and trigeminal neuralgia measured with 1H-MRS. Neurol Res. 2008;30(3):229-233.

14. Sarchielli P, Tarducci R, Presciutti O, et al. Functional 1H-MRS finding in migraine patients with and without aura assessed interictally. Neuroimage. 2005;24(4):1025-1031.

15. Reyngoudt H, Achten E, Paemeleire K. Magnetic resonance spectroscopy in migraine: what have we learned so far? Cephalalgia. 2012;32(11):845-859.

16. Harris RE, Sundgren PC, Pang Y, et al. Dynamic levels of glutamate within the insula are associated with improvements in multiple pain domain in fibromyalgia. Arthritis Rheum. 2008;58(3):903-907.

17. Zhang H, Peng Y, Liu Z, et al. Effects of acupuncture therapy on abdominal fat and hepatic fat content in obese children: a magnetic resonance imaging and proton magnetic resonance spectroscopy study. J Altern Complement Med. 2011;17(5):413-420.

18. Broner SW, Bobker S, Klebanoff L. Migraine in women. Semin Neurol. 2017;37(6):601-610.

19. State Administration of Traditional Chinese Medicine. The Criterion of Diagnosis and Therapeutic Effects of Disease and Syndrome in Traditional Chinese Medicine. Nanjing: Nanjing University Press, 1994:22-23 (ZY/T001.1-94).

20. Antonaci F, Bono G, Chimento P. Diagnosing cervicogenic headache. J Headache Pain. 2006;7(3):145-148.

21. Yang XC, Zhou YM, Lv M, et al. Clinical observation on the treatment of cervicogenic headache by muscular acupuncture stimulation therapy. Zhen Ci Yan Jiu. 2009;34:72.

22. France S, Bown J, Nowosilskyi M, Mott M, Rand S, Walters J. Evidence for the use of dry needling and physiotherapy in the management of cervicogenic or tension-type headache: a systematic review. Cephalalgia. 2014;34(12):994-1003.

23. Zheng H, Chen M, Wu X, Li Y, Liang FR. Manage migraine with acupuncture: a review of acupuncture protocols in randomized controlled trials. Am J Chin Med. 2010;38(4):639-650.

24. Le Bars D, Willer J-C, de Broucker T, Villanueva L. Neurophysiological mechanisms involved in pain-relieving effect of counter irritation and related techniques including acupuncture. In: Pomeranz B, Stux G, editors. Scientific Bases of Acupuncture. First edition. Berlin: Springer; 1989: 79-112. 
25. Niddam DM, Yeh TC, Wu YT, et al. Event-related functional MRI study on central representation of acute muscle pain induced by electrical stimulation. Neuroimage. 2002;17(3):1437-1450.

26. Wang Y, Li D, Bao F, et al. Thalamic metabolic alterations with cognitive dysfunction in idiopathic trigeminal neuralgia: a multivoxel spectroscopy study. Neuroradiology. 2014;56(8):685-693.

27. Zhang N, Song X, Bartha R, et al. Advances in high-field magnetic resonance spectroscopy in Alzheimer's disease. Curr Alzheimer Res. 2014;11(4):367-388.

28. Bollen KA, Jackman RW. Regression diagnostics: an expository treatment of outliers and influential cases. In: Fox J, Long J, editors. Modern Methods of Data Analysis. First edition. Newbury Park, CA: Sage; 1990:257-291.

29. Cohen J. Statistical Power Analysis for the Behavioral Sciences, 2nd ed. Hillsdale, NJ: Lawrence Erlbaum Associates; 1988:109-143.

30. Sawilowsky S. New effect size rules of thumb. J Mod Appl Stat Meth. 2009;8(2):597-599.

31. Pascual J, Mateos V, Roig C, Sanchez-Del-Rio M, Jiménez D. Marketed oral triptans in the acute treatment of migraine: a systematic review on efficacy and tolerability. Headache. 2007;47(8):1152-1168.

32. Wang LP, Zhang XZ, Guo J, et al. Efficacy of acupuncture for acute migraine attack: a multicenter single blinded, randomized controlled trial. Pain Med. 2012;13(5):623-630.

33. Li Y, Liang F, Yang X, et al. Acupuncture for treating acute attacks of migraine: a randomized controlled trial. Headache. 2009;49(6):805-816.

34. May A. New insights into headache: an update on functional and structural imaging findings. Nat Rev Neurol. 2009;5(4):199-209.
35. Ellerbrock I, Engel AK, May A. Microstructural and network abnormalities in headache. Curr Opin Neurol. 2013;26(4):353-359.

36. Younis S, Hougaard A, Vestergaard MB, Larsson HBW, Ashina M. Migraine and magnetic resonance spectroscopy: a systematic review. Curr Opin Neurol. 2017;30(3):246-262.

37. De Tommaso M, Ceci E, Pica C, et al. Serum levels of N-acetylaspartate in migraine and tension-type headache. $J$ Headache Pain. 2012;13(5):389-394.

38. Clark JB. N-acetyl-aspartate: a marker for neuronal loss or mitochondrial dysfunction. Dev Neurosci. 1998;20(4-5):271-276.

39. Tang JS, Qu CL, Huo FQ. The thalamic nucleus submedius and ventrolateral orbital cortex are involved in nociceptive modulation: a novel pain modulation pathway. Prog Neurobiol. 2009;89(4):383-389.

40. Eylers VV, Maudsley AA, Bronzlik P, Dellani PR, Lanfermann H, Ding XQ. Detection of normal aging effects on human brain metabolite concentrations and microstructure with whole-brain MR spectroscopic imaging and quantitative MR imaging. Am J Neuroradiol. 2016;37(3):447-454.

41. Liu G, Ma HJ, Hu PP, et al. Effects of painful stimulation and acupuncture on attention networks in healthy subjects. Behav Brain Funct. 2013;9:23.

42. Rushworth MF, Behrens TE, Rudebeck PH, Walton ME. Contrasting roles for cingulate and orbitofrontal cortex in decisions and social behaviour. Trends Cogn Sci. 2007;11(4):168-176.

43. Lim SH, Dinner DS, Pillay PK, et al. Functional anatomy of the human supplementary sensorimotor area: results of extraoperative electrical stimulation. Electroencephalogr Clin Neurophysiol. 1994;91(3):179-193.
Journal of Pain Research

\section{Publish your work in this journal}

The Journal of Pain Research is an international, peer reviewed, open access, online journal that welcomes laboratory and clinical findings in the fields of pain research and the prevention and management of pain. Original research, reviews, symposium reports, hypothesis formation and commentaries are all considered for publication.

\section{Dovepress}

The manuscript management system is completely online and includes a very quick and fair peer-review system, which is all easy to use. Visit http://www.dovepress.com/testimonials.php to read real quotes from published authors. 